\title{
Ácaros de cafeeiro (Coffea spp.) no Estado de São Paulo, Brasil. Parte II. Prostigmata
}

\author{
Jeferson Luiz de Carvalho Mineiro ${ }^{1,2}$, Adalton Raga ${ }^{1}$, Mário Eidi Sato ${ }^{1}$, \\ André Luiz Matioli ${ }^{1} \&$ Luiz Henrique Chorfi Berton ${ }^{1}$ \\ ${ }^{1}$ Laboratório de Entomologia Econômica, Centro Experimental do Instituto Biológico, \\ Rod. Heitor Penteado, Km 3, CEP 13092-543, Campinas, SP, Brasil \\ ${ }^{2}$ Autor correspondência: Jeferson Luiz de Carvalho Mineiro, e-mail: jefmin@hotmail.com
}

MINEIRO, J.L.C., RAGA, A., SATO, M.E., MATIOLI, A.L. \& BERTON, L.H.C. Mites of coffee plants (Coffea spp.) in State of São Paulo. Part II. Prostigmata. Biota Neotrop. 10(4): http://www.biotaneotropica. org.br/v10n4/en/abstract?article+bn04010042010.

\begin{abstract}
The objective of this study was to carry out a survey of mite species belonging to the suborder Prostigmata on Coffea spp. in different localities of the State of São Paulo. Samples of coffee (Coffea spp.) leaves and branches were collected in 24 municipalities of the State of São Paulo, in different periods of the year. All mites collected were mounted on microscope slides. Whenever possible, mites were identified to species level. Seventy-nine species were identified, belonging to the families Anystidae, Bdellidae, Cheyletidae, Cunaxidae, Diptilomiopidae, Eriophyidae, Eupalopsellidae, Eupodidae, Iolinidae, Meyerellidae, Nanorchestidae, Paratydeidae, Raphignathidae, Stigmaeidae, Tarsonemidae, Tenuipalpidae, Tetranychidae, Trombidiidae, Tuckerellidae e Tydeidae.

Keywords: Acari, diversity of mite, predaceous mites, phytophagus mites.
\end{abstract}

MINEIRO, J.L.C., RAGA, A., SATO, M.E., MATIOLI, A.L. \& BERTON, L.H.C. Ácaros de cafeeiro (Coffea spp.) no Estado de São Paulo, Brasil. Parte II. Prostigmata. Biota Neotrop. 10(4): http://www.biotaneotropica.org. br/v10n4/pt/abstract?article+bn04010042010.

Resumo: Este trabalho teve como objetivo fazer um levantamento das espécies de ácaros pertencentes à subordem Prostigmata em Coffea spp. em diferentes localidades do Estado de São Paulo. Folhas e ramos de cafeeiros (Coffea spp.) foram coletados em 24 municípios do Estado de São Paulo e em diferentes épocas do ano. Todos os ácaros encontrados foram montados em lâminas de microscopia. A identificação dos ácaros foi feita até o nível específico quando possível. Foram identificadas 79 espécies de ácaros pertencentes às famílias Anystidae, Bdellidae, Cheyletidae, Cunaxidae, Diptilomiopidae, Eriophyidae, Eupalopsellidae, Eupodidae, Iolinidae, Meyerellidae, Nanorchestidae, Paratydeidae, Raphignathidae, Stigmaeidae, Tarsonemidae, Tenuipalpidae, Tetranychidae, Trombidiidae, Tuckerellidae e Tydeidae.

Palavras-chave: Acari, diversidade de ácaros, ácaros predadores, ácaros fitófagos. 


\section{Introdução}

Dos organismos encontrados nesta cultura, destacam-se algumas espécies de ácaros fitófagos que podem causar prejuízos, e outras de predadores que também podem estar atuando sobre estas. A subordem Prostigmata é o grupo mais heterogêneo dentro de Acari. Os ácaros fitófagos e algumas espécies de predadores mais comumente encontrados sobre plantas pertencem a esta ordem (Mineiro et al. 2006a, b, Moraes \& Flechtmann 2008).

Dentre os ácaros fitófagos encontrados em Coffea spp. no Brasil, os mais conhecidos são os representantes das famílias Tetranychidae e Tenuipalpidae (Calza \& Sauer 1952, Flechtmann 1967, Heinrich 1972, Pallini Filho et al. 1992, Reis et al. 2000, Mineiro et al. 2001, 2006a, b, 2008a, Spongoski et al. 2005, Reis \& Zacarias 2007, Franco et al. 2008).

Este trabalho teve como objetivo fazer um levantamento das espécies de ácaros pertencentes à subordem Prostigmata em cafeeiros (Coffea spp.) em diferentes localidades do Estado de São Paulo.

\section{Material e Métodos}

O levantamento foi realizado em 24 municípios do Estado de São Paulo, entre 2001 e 2008 (Tabela 1). Neste manuscrito também foram incluídas as espécies já relatadas para os municípios de Garça e Jeriquara (Mineiro et al. 2006a). O material (folhas e ramos) foi coletado em plantios comerciais e não comercias. As folhas e ramos foram retirados ao acaso do terço médio das plantas. Foram coletadas 10 folhas e dois ramos de cada planta. O material foi colocado em sacos plásticos, acondicionados em caixas de isopor e transportados para o Laboratório de Entomologia Econômica do Instituto Biológico em Campinas, SP (LEE-IB). Para a retirada dos ácaros, as folhas foram mergulhadas em recipiente plástico contendo álcool 70\%. Após 10 minutos, as folhas foram agitadas nesta solução para que os ácaros presentes ficassem no líquido do recipiente. Em seguida, esta solução foi passada em peneira com malha de $0,038 \mathrm{~mm}$ e o material retido foi transferido para um frasco de vidro para posterior triagem. Os ácaros coletados foram montados em lâminas de microscopia para posterior identificação até espécie, quando possível. Para exame ao microscópio óptico com contraste de fases, os eriofiídeos foram montados em meio modificado de Berlese (Jeppson et al. 1975) e os demais montados em meio de Hoyer (Walter \& Krantz 2009).

Uma amostra representativa dos espécimes encontrados está depositada na coleção de referência de ácaros "Geraldo Calcagnolo" do LEE-IB.

\section{Resultados}

Foram identificadas espécies de ácaros pertencentes às famílias Anystidae, Bdellidae, Cheyletidae, Cunaxidae, Diptilomiopidae, Eriophyidae, Eupalopsellidae, Eupodidae, Iolinidae, Meyerellidae, Nanorchestidae, Paratydeidae, Raphignathidae, Stigmaeidae, Tarsonemidae, Tenuipalpidae, Tetranychidae, Trombidiidae, Tuckerellidae e Tydeidae. ácaros.

São apresentadas a seguir, as famílias, gêneros e espécies de

\section{ANYSTIDAE OUDEMANS, 1902}

Erythracarus nasutus Otto, 1999

Erythracarus nasutus Otto, 1999: 844.

Espécime examinado: 1 , Atibaia, Sítio Kurosawa, C. arabica cv. Mundo Novo, 22.i.2008.
BDELLIDAE DUGÈS, 1834

Bdella ueckermanni Hernandes, Daud \& Feres, 2008

Bdella ueckermanni Hernandes, Daud \& Feres, 2008: 262.

Espécimes examinados: 3 + , Jeriquara, Fazenda Boa Esperança, C. arabica cv. Mundo Novo, 11.vii.2001; 17.xii.2001 e 04.ii.2002; 18 , Atibaia, Sítio Kurosawa, C. arabica cv. Mundo Novo, 26.iii.2007, 21.v.2007, 23.vii.2007, 24.ix.2007, 22.x.2007 e 14.vii.2008; cv. Catuaí Amarelo, 27.vi.2000.

Hexabdella cinquaginta Hernandes, Daud \& Feres, 2007

Hexabdella cinquaginta Hernandes, Daud \& Feres, 2007: 60.

Espécimes examinados: 21 , Jeriquara, Fazenda Boa Esperança, C. arabica cv. Mundo Novo, 04.iv.2001, 06.x.2001, 06.v.2002, 27.ix.2002, 29.iii.2003, 12.iv.2003, 26.iv.2003, 06.vi.2003 e 20.vi.2003; Fazenda São Francisco, C. arabica cv. Mundo Novo, 08.iii.2002; 5q, Garça, Estação Experimental Alcides Carvalho, C. arabica cv. Mundo Novo, 05.iv.2001, 17.i.2002, 21.ix.2001, 06.vii.2001 e 15.v.2003; 24, Atibaia, Sítio Kurosawa, C. arabica cv. Catuaí Amarelo, 25.vii.2000; C. arabica cv. Mundo Novo, 22.ii.2007, 26.iii.2007, 26.iii.2007, 23.iv.2008, 16.ix.2008 e 10.xi.2008; 7 , Monte Alegre do Sul, Sítio São José, C. arabica cv. Mundo Novo, 01.iv.2004, 29.vii.2004, 16.iii.2005, 03.v.2005, 08.vii.2005, 14.vi.2006 e 18.ix.2007.

Tabela 1. Coordenadas geográficas dos municípios no Estado de São Paulo onde foram realizadas as coletas.

Table 1. Geographic coordinates of the municipalities in the State of São Paulo where the mites were collected.

\begin{tabular}{|c|c|c|}
\hline Município & Coordenadas & Altitude (m) \\
\hline Assis & $22^{\circ} 39^{\prime} 39^{\prime \prime} \mathrm{S}, 50^{\circ} 25^{\prime} 13^{\prime \prime} \mathrm{W}$ & 560 \\
\hline Atibaia & $23^{\circ} 06^{\prime} 59^{\prime \prime} \mathrm{S}, 46^{\circ} 33^{\prime} 06^{\prime \prime} \mathrm{W}$ & 800 \\
\hline Campinas & $22^{\circ} 53^{\prime} 20^{\prime \prime} \mathrm{S}, 47^{\circ} 04^{\prime} 40^{\prime \prime} \mathrm{W}$ & 680 \\
\hline Cândido Mota & $22^{\circ} 44^{\prime} 52^{\prime \prime} \mathrm{S}, 50^{\circ} 23^{\prime} 08^{\prime \prime} \mathrm{W}$ & 460 \\
\hline Dois Córregos & $22^{\circ} 21^{\prime} 53^{\prime \prime} \mathrm{S}, 48^{\circ} 22^{\prime} 50^{\prime \prime} \mathrm{W}$ & 677 \\
\hline Franca & $20^{\circ} 32^{\prime} 18^{\prime \prime} \mathrm{S}, 47^{\circ} 24^{\prime} 06^{\prime \prime} \mathrm{W}$ & 1000 \\
\hline Garça & $22^{\circ} 12^{\prime} 55^{\prime \prime} \mathrm{S}, 49^{\circ} 39^{\prime} 04^{\prime \prime} \mathrm{W}$ & 682 \\
\hline Itirapuã & $20^{\circ} 38^{\prime} 30^{\prime \prime} \mathrm{S}, 47^{\circ} 13^{\prime} 09^{\prime \prime} \mathrm{W}$ & 860 \\
\hline Itupeva & $23^{\circ} 09^{\prime} 12^{\prime \prime} \mathrm{S}, 47^{\circ} 03^{\prime} 28^{\prime \prime} \mathrm{W}$ & 675 \\
\hline Jaboticabal & $21^{\circ} 15^{\prime} 20^{\prime \prime} \mathrm{S}, 48^{\circ} 19^{\prime} 16^{\prime \prime} \mathrm{W}$ & 583 \\
\hline Jaguariúna & $22^{\circ} 42^{\prime} 18^{\prime \prime} \mathrm{S}, 46^{\circ} 59^{\prime} 22^{\prime \prime} \mathrm{W}$ & 580 \\
\hline Jeriquara & $20^{\circ} 18^{\prime} 42^{\prime \prime} \mathrm{S}, 47^{\circ} 35^{\prime} 20^{\prime \prime} \mathrm{W}$ & 860 \\
\hline Lutécia & $22^{\circ} 20^{\prime} 24^{\prime \prime} \mathrm{S}, 50^{\circ} 23^{\prime} 32^{\prime \prime} \mathrm{W}$ & 580 \\
\hline Maracaí & $22^{\circ} 36^{\prime} 39^{\prime \prime} \mathrm{S}, 50^{\circ} 40^{\prime} 01^{\prime \prime} \mathrm{W}$ & 380 \\
\hline Mococa & $21^{\circ} 28^{\prime} 16^{\prime \prime} \mathrm{S}, 47^{\circ} 00^{\prime} 23^{\prime \prime} \mathrm{W}$ & 620 \\
\hline Monte Alegre do Sul & $22^{\circ} 40^{\prime} 50^{\prime \prime} \mathrm{S}, 46^{\circ} 40^{\prime} 45^{\prime \prime} \mathrm{W}$ & 760 \\
\hline Monte Mor & $22^{\circ} 56^{\prime} 47^{\prime \prime} \mathrm{S}, 47^{\circ} 18^{\prime} 58^{\prime \prime} \mathrm{W}$ & 560 \\
\hline Olímpia & $20^{\circ} 44^{\prime} 16^{\prime \prime} \mathrm{S}, 48^{\circ} 55^{\prime} 02^{\prime \prime} \mathrm{W}$ & 500 \\
\hline Paranapuã & $20^{\circ} 06^{\prime} 15^{\prime \prime} \mathrm{S}, 50^{\circ} 35^{\prime} 12^{\prime \prime} \mathrm{W}$ & 600 \\
\hline Pindamonhangaba & $22^{\circ} 55^{\prime} 33^{\prime \prime} \mathrm{S}, 45^{\circ} 27^{\prime} 40^{\prime \prime} \mathrm{W}$ & 540 \\
\hline Piracicaba & $22^{\circ} 42^{\prime} 30^{\prime \prime} \mathrm{S}, 47^{\circ} 38^{\prime} 01^{\prime \prime} \mathrm{W}$ & 554 \\
\hline São Paulo & $23^{\circ} 32^{\prime} 52^{\prime \prime} \mathrm{S}, 46^{\circ} 38^{\prime} 07^{\prime \prime} \mathrm{W}$ & 860 \\
\hline Tatuí & $23^{\circ} 21^{\prime} 03^{\prime \prime} \mathrm{S}, 47^{\circ} 50^{\prime} 53^{\prime \prime} \mathrm{W}$ & 620 \\
\hline Tietê & $23^{\circ} 06^{\prime} 03^{\prime \prime} \mathrm{S}, 47^{\circ} 42^{\prime} 55^{\prime \prime} \mathrm{W}$ & 500 \\
\hline
\end{tabular}


Spinibdella denheyeri Hernandes, Daud \& Feres, 2008

Spinibdella denheyeri Hernandes, Daud \& Feres, 2008: 265.

Espécimes examinados: 6엉 Jeriquara, Fazenda Boa Esperança, C. arabica cv. Mundo Novo, 06.x.2001, 19; 03.xi.2001, 28.ii.2002, 02.ix.2002 e 16.ii.2003; Fazenda São Francisco, C. arabica cv. Catuaí Amarelo, 17.i.2003; 3 , Monte Alegre do Sul, Sítio São José, C. arabica cv. Mundo Novo, 02.ii.2005, 09.iii.2005 e 16.iii.2005; 1 , , Atibaia, Sítio Kurosawa, C. arabica cv. Mundo Novo, 14.vii.2008.

\section{Spinibdella sp.1}

Espécimes examinados: 2 , Garça, Estação Experimental Alcides Carvalho, Garcafé, C. arabica cv. Mundo Novo, 25.x.2001, 06.xi.2001 e 03.x.2002; 2 엉 Jeriquara, Fazenda Boa Esperança, C. arabica cv. Mundo Novo, 18.iv.2001 e 16.v.2001.

\section{Spinibdella sp.2}

Espécime examinado: 1 , Monte Alegre do Sul, Sítio São José, C. arabica cv. Mundo Novo, 22.iv.2004.

\section{Spinibdella sp.3}

Espécimes examinados: 1 , Paranapuã, C. arabica cv. Apoatã, 25.iii.2004; 3 , Monte Alegre do Sul, Sítio São José, C. arabica cv. Mundo Novo, 12.v.2004, 05.x.2004 e 05.x.2005; 1 , Jeriquara, Fazenda Boa Esperança, C. arabica cv. Mundo Novo, 01.xii.2001.

Tetrabdella sp.

Espécime examinado: 1 , Atibaia, C. arabica cv. Mundo Novo, 25.vi.2007.

\section{CHEYLETIDAE LEACH, 1815}

\section{Cheletogenes sp.}

Espécimes examinados: 1\%, Garça, Estação Experimental Alcides Carvalho, Garcafé, C. arabica cv. Icatu Vermelho, 08.vi.2001; 1올 Jeriquara, Fazenda São Francisco, C. arabica cv. Catuaí Amarelo, 15.v.2003; 1ㅇ, Monte Alegre do Sul, Sítio São José, C. arabica cv. Mundo Novo, 09.iii.2007.

\section{Cheletomimus sp.}

Espécimes examinados: 2 + , Jeriquara, Fazenda Boa Esperança, C. arabica cv. Mundo Novo, 09.viii.2001; Fazenda São Francisco, C. arabica cv. Catuaí Amarelo, 17.iv.2003; 1q, Monte Alegre do Sul, Sítio São José, C. arabica cv. Mundo Novo, 14.vi.2006; 1 9 , Atibaia, Sítio Kurosawa, C. arabica cv. Mundo Novo, 25.vi.2007.

Cheletomorpha sp.

Espécime examinado: $1+$ Jeriquara, Fazenda São Francisco, C. arabica cv. Catuaí Amarelo, 20.iii.2003.

\section{Cheyletus sp.}

Espécimes examinados: 2 + , Garça, Estação Experimental Alcides Carvalho, Garcafé, C. arabica cv. Mundo Novo, 05.iv.2001 e 05.ix.2001; 1 q, Jeriquara, Fazenda Boa Esperança, C. arabica cv. Mundo Novo, 09.v.2003.

Chiapocheylus sp.

Espécimes examinados: 3 , Jeriquara, Fazenda Boa Esperança, C. arabica cv. Mundo Novo, 12.iv.2003 e 09.v.2003.
Eucheyletia sp.

Espécimes examinados: 2 , Garça, Estação Experimental Alcides Carvalho, Garcafé, C. arabica cv. Mundo Novo, 23.vii.2001 e 16.iv.2003; 2 , Jeriquara, Fazenda Boa Esperança, C. arabica cv. Mundo Novo, 30.v.2001 e 20.vi.2003.

Grallacheles sp.

Espécimes examinados: 7q, Garça, Estação Experimental Alcides Carvalho, Garcafé, C. arabica cv. Icatu Amarelo, 02.iii.2003; cv. Catuaí Amarelo, 03.vii.2002 e 02.iii.2003; cv. Mundo Novo, 03.vii.2002, 18.vii.2002, 16.viii.2002 e 02.iii.2003; C. canephora cv. Apoatã, 02.iv.2002; 1 , Jeriquara, Fazenda Boa Esperança, C. arabica cv. Mundo Novo, 06.vi.2003.

\section{Hemicheyletia sp.}

Espécimes examinados: 2q, Garça, Estação Experimental Alcides Carvalho, Garcafé, C. arabica cv. Icatu Vermelho, 06.vii.2001; 5 + , Jeriquara, Fazenda Boa Esperança, C. arabica cv. Mundo Novo, 04.iv.2001; 22.ix.2001; 20.x.2001 e 17.xi.2001.

Prosocheyla sp.

Espécimes examinados: 6ㅇ, Atibaia, Sítio Kurosawa, C. arabica cv. Catuaí Amarelo, 27.vi.2000, 11.vii.2000 e 25.vii.2000; 1 , , Jeriquara, Fazenda Boa Esperança, C. arabica cv. Mundo Novo, 02.ix.2002; 1 + , Monte Alegre do Sul, Sítio São José, C. arabica cv. Mundo Novo, 14.vi.2006.

\section{CUNAXIDAE THOR, 1902}

\section{Armascirus sp.1}

Espécimes examinados: 1 \% , Jeriquara, Fazenda Boa Esperança, C. arabica cv. Mundo Novo, 21.i.2002; 15, Monte Alegre do Sul, Sítio São José, C. arabica cv. Mundo Novo, 22.iv.2004 e 28.iv.2004.

Armascirus sp. 2

Espécimes examinados: 9, Monte Alegre do Sul, Sítio São José, C. arabica cv. Mundo Novo, 14.i.2005, 02.ii.2005 e 02.iii.2005.

\section{Armascirus sp. 3}

Espécime examinado: 1 , Monte Alegre do Sul, Sítio São José, C. arabica cv. Mundo Novo, 01.iv.2004.

Dactyloscirus sp.

Espécimes examinados: 3 , Jeriquara, Fazenda Boa Esperança, C. arabica cv. Mundo Novo, 24.viii.2001; Fazenda São Francisco, cv. Catuaí Amarelo, 20.iii.2003 e 17.iv.2003; 2 \&, Garça, Estação Experimental Alcides Carvalho, C. arabica cv. Icatu Vermelho, 10.xii.2001 e cv. Mundo Novo, 02.iii.2003; 5, Monte Alegre do Sul, sítio São José, C. arabica cv. Mundo Novo, 01.iv.2004, 23.xii.2004 e 02.ii.2005.

Neocunaxoides $\mathrm{sp}$.

Espécimes examinados: 39 , Garça, Estação Experimental Alcides Carvalho, Garcafé, C. arabica cv. Icatu Amarelo, 03.i.2002; C. canephora cv. Apoatã, 05.ix.2001.

\section{DIPTILOMIOPIDAE KEIFER, 1944}

Catarhinus tricholaenae Keifer, 1959

Catarhinus tricholaenae Keifer, 1959: 11; Flechtmann \& Aranda, 1970: 94; Flechtmann \& Santana, 1997: 189.

Espécimes examinados: 3 , Garça, Estação Experimental Alcides Carvalho, C. arabica cv. Mundo Novo, 30.xii.2002; 
C. canephora cv. Apoatã, 30.xii.2002; 18 , Jeriquara, Fazenda Boa Esperança, C. arabica cv. Mundo Novo, 21.i.2002, 16.ii.2002, 04.vii.2002, 07.i.2003, 18.i.2003, 31.i.2003, 16.ii.2003; 6우, Fazenda São Francisco, C. arabica cv. Mundo Novo, 17.i.2003, 15.v.2003; 8ㅇ, Monte Alegre do Sul, Sítio São José, C. arabica cv. Mundo Novo, 01.iv.2004, 13.iv.2004, 23.xii.2004, 30.xii.2004, 14.i.2005, 02.iii.2005 e 09.iii.2005.

Relatos prévios no Brasil: São Paulo (Keifer 1959, Flechtmann \& Aranda 1970, Flechtmann \& Santana 1997).

Observação: No Brasil, C. tricholaenae é encontrado em gramíneas, Tricholaena rosea e em Zea mays L. (Flechtmann \& Aranda, 1970).

\section{ERIOPHYIDAE NALEPA, 1898}

\section{Aceria sp.}

Espécimes examinados: 4 ㅇ, Jeriquara, Fazenda Boa Esperança, C. arabica cv. Mundo Novo, 14.ix.2002, 28.ii.2003 e 13.iv.2003; Fazenda São Francisco, C. arabica cv. Mundo Novo, 17.i.2003; 1 , Garça, Estação Experimental Alcides Carvalho, C. arabica, 01.ii.2002; 8 , , Monte Alegre do Sul, Sítio São José, C. arabica cv. Mundo Novo, 23.iv.2004, 30.xii.2004, 09.iii.2005, 14.i.2005, 16.iii.2005 e 05.x.2005.

Eriophyes sp.

Espécime examinado: 1 , Jeriquara, Fazenda Boa Esperança, C. arabica cv. Mundo Novo, 02.v.2001.

\section{Phyllocoptruta sp.}

Espécimes examinados: $3 q$, Monte Alegre do Sul, Sítio São José, C. arabica cv. Mundo Novo, 07.vi.2004, 09.iii.2005 e 16.iii.2005; 45 , Atibaia, Sítio Kurosawa, C. arabica cv. Mundo Novo, 22.ii.2007 e 20.viii.2007; 4 , Garça, Estação Experimental Alcides Carvalho, C. arabica cv. Mundo Novo, 30.iv.2003, 20.xi.2002 e 06.iv.2001; 22 , Jeriquara, Fazenda Boa Esperança, C. arabica cv. Mundo Novo, 02.v.2001, 16.v.2001, 30.v.2001, 05.i.2002, 28.iii.2002, 30.iv.2002, 19.ii.2002, 15.iii.2003, 12.iv.2003, 26.iv.2003 e 29.iii.2003.

Shevtchenkella sp.

Espécimes examinados: 3 + , Jeriquara, Fazenda Boa Esperança, C. arabica cv. Mundo Novo, 12.iv.2002; Fazenda São Francisco, C. arabica cv. Mundo Novo, 17.i.2003; 2ᄋ, Garça, Estação Experimental Alcides Carvalho, C. arabica, 05.iv.2002 e 01.ii.2002.

\section{EUPALOPSELLIDAE WILLMANN, 1952}

\section{Eupalopsellus sp.}

Espécimes examinados: 2 + , Jeriquara, Fazenda Boa Esperança, C. arabica cv. Mundo Novo, 26.x.2002 e 18.i.2003; 1 , , Fazenda São Francisco, C. arabica cv. Catuaí Amarelo, 20.iii.2003, 1 .

Exothorhis caudata Summers, 1960

Exothorhis caudata Summers, 1960: 131; Matioli et al., 2002: 116.

Exothorhis citri Meyer \& Ueckermann, 1989: 10, nova sinonímia segundo Matioli et al. 2002.

Espécimes examinados: 1 , , Jaboticabal, UNESP/FCAV, C. arabica, 18.vii.2003; 1ㅇ, Monte Mor, Faz. Mundo Novo, C. arabica cv. Mundo Novo, 01.ix.2005; 19, Garça, Estação Experimental Alcides Carvalho, C. arabica cv. Mundo Novo, 01.iv.2002; 1 + , Monte Alegre do Sul, Sítio São José, C. arabica cv. Mundo Novo, 23.iii.2005.

Relatos prévios no Brasil: São Paulo (Matioli et al. 2002, Vis et al. 2006).
Exothorhis sp.

Espécimes examinados: 2 , Atibaia, Sítio Kurosawa, C. arabica cv. Catuaí Amarelo, 27.vi.2000 e 11.vii.2000; $15 q$ e 2n, Garça, Estação Experimental Alcides Carvalho, Garcafé, C. arabica cv. Mundo Novo, 03.v.2002; 18.vii.2002, 18.iii.2003, 02.iv.2003 e 16.iv.2003; cv. Icatu Amarelo, 03.i.2002; C. canephora cv. Apoatã, 03.vii.2002; 7 , Jeriquara, Fazenda Boa Esperança, C. arabica cv. Mundo Novo, 02.v.2001, 13.vi.2001, 11.vii.2001, 12.iv.2002 e 30.iv.2002; 19, Maracaí, Sítio Santa Margarida, C. arabica, 31.v.2006; 2 + , Monte Alegre do Sul, Sítio São José, C. arabica cv. Mundo Novo, 14.vi.2006.

\section{EUPODIDAE KOCH, 1842}

\section{Eupodes sp.}

Espécimes examinados: 39 , Atibaia, Sítio Kurozawa, C. arabica cv. Catuaí Amarelo, 27.vi.2000, 11.vii.2000 e 25.vii.2000; 12 q , Jeriquara, Fazenda Boa Esperança, C. arabica cv. Mundo Novo, 25.v.2002, 03.xi.2001, 18.iv.2001, 30.iv.2002, 18.i.2003, 07.i.2003, 16.ii.2003, 12.iv.2003 e 20.vi.2006; Fazenda São Francisco, C. arabica cv. Catuaí Amarelo, 20.iii.2003 e 20.vi.2003; 1 , , Dois Córregos, C. arabica, 07.xi.2003; 4우, Garça, Estação Experimental Alcides Carvalho, C. canephora cv. Apoatã, 06.xi.2001 e 04.x.2002, C. arabica cv. Mundo Novo, 17.v.2002 e 17.ix.2002; 1 + , Monte Alegre do Sul, Sítio São José, C. arabica cv. Mundo Novo, 12.v.2004.

\section{IOLINIDAE PRITCHARD, 1956}

Homeopronematus sp.

Espécimes examinados: 2 , Jeriquara, Fazenda Boa Esperança, C. arabica cv. Mundo Novo, 16.v.2001, e 14.x.2002; 4ㅇ, Garça, Estação Experimental Alcides Carvalho, C. arabica cv. Mundo Novo, 05.iv.2001; cv. Icatu Vermelho, 05.iv.2001; 1q, Monte Alegre do Sul, Sítio São José, C. arabica cv. Mundo Novo, 12.xii.2007.

Parapronematus acaciae Baker, 1965

Parapronematus acaciae Baker, 1965; Zacarias, 2001:74.

Espécimes examinados: 1 \% , Tietê, CATI, C. arabica cv. Obatã, 07.viii.2003; 1 , Pindamonhangaba, Fazenda Santa Emília, C. arabica, 04.i.2004; 1ㅇ, Atibaia, Sítio Kurosawa, 27.vi.2000; 20 , Jeriquara, Fazenda Boa Esperança, C. arabica cv. Mundo Novo, 06.vi.2003; Fazenda São Francisco, 03.iii.2002, 23.iii.2002, 06.v.2002 e 08.vi.2002; 4ㅇ, Monte Alegre do Sul, Sítio São José, C. arabica cv. Mundo Novo, 16.xii.2004 e 14.vi.2006; 2 , , São Paulo, IB, C. arabica cv. Mundo Novo, 27.ii.2008.

Relatos prévios no Brasil: São Paulo (Mineiro \& Raga 2003, Vis et al. 2006, Mineiro et al. 2006a, b).

\section{MEYERELLIDAE ANDRÉ, 1979}

Triophtydeus sp.

Espécime examinado: 1 , Jeriquara, Fazenda Boa Esperança, C. arabica cv. Mundo Novo, 11.vii.2001.

\section{NANORCHESTIDAE GRANDJEAN, 1937}

\section{Nanorchestes sp.}

Espécimes examinados: 3 , Garça, Estação Experimental Alcides Carvalho, C. arabica cv. Mundo Novo, 06.ix.2002; cv. Icatu Amarelo, 29.i.2003 e C. canephora cv. Apoatã, 07.viii.2001; 5 ㅇ, Jeriquara, Fazenda Boa Esperança, C. arabica cv. Mundo Novo, 16.v.2001, 11.vii.2001, 25.vii.2001 e 06.x.2001. 
Speleorchestes $\mathrm{sp}$.

Espécimes examinados: 3 \% , Garça, Estação Experimental Alcides Carvalho, C. arabica cv. Mundo Novo, 17.x.2001; cv. Icatu Amarelo, 10.x.2001 e C. canephora cv. Apoatã, 03.i.2002; 9 , Jeriquara, Fazenda Boa Esperança, C. arabica cv. Mundo Novo, 25.vii.2001, 21.vi.2002, 04.vii.2002, 18.viii.2002, 02.ix.2002 e 31.i.2003; Fazenda São Francisco, C. arabica cv. Catuai Amarelo, 15.v.2003.

\section{PARATYDEIDAE BAKER, 1949}

\section{Neotydeus sp.}

Espécimes examinados: 1\%, Garça, Estação Experimental Alcides Carvalho, C. arabica cv. Mundo Novo, 16.iv.2003.

Sacotydeus sp.

Espécime examinado: 1 엉 Jeriquara, Fazenda Boa Esperança, C. arabica cv. Mundo Novo, 17.xii.2001.

Scolotydeus sp.

Espécimes examinados: 2 , Garça, Estação Experimental Alcides Carvalho, C. arabica cv. Mundo Novo, 18.vi.2002 e C. canephora cv. Apoatã, 07.xi.2002; 1 \% , Jeriquara, Fazenda Boa Esperança, C. arabica cv. Mundo Novo, 12.iv.2002.

Tanytydeus sp.

Espécime examinado: 1 , Jeriquara, Fazenda Boa Esperança, C. arabica cv. Mundo Novo, 17.xi.2001.

\section{RAPHIGNATHIDAE KRAMER, 1877}

\section{Raphignathus sp.}

Espécimes examinados: 1 , , Atibaia, Sítio Kurosawa, C. arabica cv. Catuaí Amarelo, 25.vii.2000; 7 9 , Jeriquara, Fazenda Boa Esperança, C. arabica cv. Mundo Novo, 04.iv.2001, 18.iv.2001, 13.vi.2001, 25.vii.2001, 01.xii.2001 e 14.x.2002; Fazenda São Francisco, C. arabica cv. Catuaí Amarelo, 19.ii.2003; 11우, Garça, Estação Experimental Alcides Carvalho, C. arabica cv. Mundo Novo, 21.xi.2001, 01.ii.2002, 15.ii.2002, 01.iv.2002 e 19.xii.2002; cv. Icatu Amarelo, 10.xii.2001 e 02.iii.2003; cv. Catuaí Amarelo, 03.x.2002; C. canephora cv. Apoatã, 04.x.2002.

\section{STIGMAEIDAE OUDEMANS, 1931}

Agistemus brasiliensis Matioli, Ueckermann \& Oliveira

Agistemus brasiliensis Matioli, Ueckermann \& Oliveira, 2002: 106.

Espécimes examinados: 5 q , Jeriquara, Fazenda Boa Esperança, C. arabica cv. Mundo Novo, 30.v.2001, 21.i.2002, 30.iv.2002 e 19.vii.2002; 12, Garça, Estação Experimental Alcides Carvalho, C. arabica cv. Icatu Vermelho, 09.v.2001, 06.vii.2001 e 03.vi.2002; cv. Catuaí Amarelo, 06.iv.2001; cv. Mundo Novo, 19.iv.2002; C. canephora cv. Apoatã, 02.iv.2002; 1 , Campinas, IAC, C. canephora cv. Guarini, 02.ix.2003; 11 , Assis, APTA Pólo Regional do Médio Paranapanema, Obatã, 05.ix.2003; 2 , Franca, Sítio Ripa, C. arabica cv. Catuaí Vermelho, 20.ii.2003; 1우 Jaguariúna, C. arabica, 08.xi.2003; 10우, Olímpia, Sítio São Pedro, C. arabica, 07.v.2004; 4, Monte Alegre do Sul, Sítio São José, C. arabica cv. Mundo Novo, 01.iv.2004, 13.iv.2004 e 21.v.2004.

Relatos prévios no Brasil: São Paulo (Matioli et al. 2002, Mineiro et al. 2006a, b).
Agistemus floridanus Gonzalez-Rodrigues, 1965

Agistemus floridanus Gonzalez-Rodrigues, 1965: 42; Arruda Filho \& Moraes, 2003: 52; Matioli et al., 2002: 103; Hernandes \& Feres 2006:7; Ferla et al. 2007: 4.

Espécimes examinados: 2 , Monte Alegre do Sul, Sítio São José, C. arabica cv. Mundo Novo, 16.iii.2006; APTA - Pólo Regional do Leste Paulista, C. arabica, 27.vii.2006.

Relatos prévios no Brasil: Rio Grande do Sul, São Paulo (Flechtmann 1981, Matioli et al. 2002, Arruda Filho \& Moraes 2003, Hernandes \& Feres 2006, Ferla et al. 2007).

Apostigmaeus sp.

Espécime examinado: 1 , Atibaia, sítio Kurosawa, C. arabica cv. Catuaí Amarelo, 27.vi.2000.

Stigmaeus sp.

Espécimes examinados: 19, Atibaia, sítio Kurosawa, C. arabica cv. Catuaí Amarelo, 11.vii.2000; 19, Garça, Estação Experimental Alcides Carvalho, C. canephora cv. Apoatã, 09.v.2001.

Zetzellia agistzellia Hernandes \& Feres, 2005

Zetzellia agistzellia Hernandes \& Feres, 2005: 28.

Espécimes examinados: 29 , Atibaia, sítio Kurosawa, C. arabica cv. Mundo Novo, 16.ii.2009.

Relatos prévios no Brasil: São Paulo (Hernandes \& Feres 2005, 2006).

Zetzellia malvinae Matioli, Ueckermann \& Oliveira

Zetzellia malvinae Matioli, Ueckermann \& Oliveira, 2002: 111.

Espécimes examinados: 6, Garça, Estação Experimental Alcides Carvalho, C. arabica cv. Icatu Amarelo, 10.xii.2001; cv. Mundo Novo, 24.viii.2001 e 13.vi.2003; cv. Catuaí Amarelo, 30.iv.2003; 21우 , Jeriquara, Fazenda Boa Esperança, C. arabica cv. Mundo Novo, 30.v.2001, 06.ix.2001, 22.ix.2001, 17.xii.2001 e 05.i.2002; 1 , , Cândito Mota, Distrito Frutal do Campo, C. arabica, 10.iii.2004; 1 9, Atibaia, sítio Kurosawa, C. arabica cv. Mundo Novo, 21.v.2007; 5, Monte Alegre do Sul, Sítio São José, C. arabica cv. Mundo Novo, 03.ix.2004; 01.iv.2004; 23.iii.2005; 20.iv.2006 e 14.vi.2006.

Relatos prévios no Brasil: São Paulo (Matioli et al. 2002, Vis et al. 2006, Mineiro et al. 2006).

TARSONEMIDAE KRAMER, 1877

\section{Daidalotarsonemus sp.}

Espécimes examinados: 4 q , Jeriquara, Fazenda Boa Esperança, C. arabica cv. Mundo Novo, 11.vii.2001, 09.viii.2001, 16.ii.2002 e 12.iv.2002; 8 , Garça, Estação Experimental Alcides Carvalho, C. arabica cv. Mundo Novo, 10.x.2001, 01.ii.2002, 03.v.2002 e 02.iii.2002; cv. Icatu Amarelo, 05.iv.2001; cv. Icatu Vermelho, 04.xii.2002.

\section{Fungitarsonemus sp.}

Espécimes examinados: 19, Garça, Estação Experimental Alcides Carvalho, C. canephora cv. Apoatã, 29.i.2003; 7 , Cândido Mota, Distrito Frutal do Campo, C. arabica cv. Catuaí Vermelho, 10.iii.2004; 7 , Jeriquara, Fazenda Boa Esperança, C. arabica cv. Mundo Novo, 11.vii.2001; Fazenda São Francisco, 15.ii.2001; 1, Jaboticabal, UNESP/FCAV, C. arabica, 18.vii.2003; 2ᄋ, Franca, sítio Ripa, C. arabica cv. Catuaí Vermelho, 20.ii.2003; 5q, Lutécia, C. arabica cv. Catuaí 
Vermelho, 10.iii.2004; 2 \%, Paranapuã, C. canephora cv. Apoatã, 25.iii.2004; 4 , , Monte Alegre do Sul, sítio São José, C. arabica cv. Mundo Novo, 01.iv.2004; 6 ㅇ, Maracaí, Sítio Santa Margarida, C. arabica, 31.v.2006; 69, Atibaia, sítio Kurosawa, C. arabica cv. Mundo Novo, 22.x.2007.

Polyphagotarsonemus latus (Banks, 1904)

Tarsonemus latus Banks, 1904: 1553.

Hemitarsonemus latus; Ewing, 1939: 54.

Neotarsonemus latus; Smiley, 1967: 137.

Polyphagotarsonemus latus (Banks), Beer \& Nucifora, 1965:42; Lindquist, 1986: 233; Feres, 2000; Hernandes \& Feres, 2006.

Espécimes examinados: 2 , Garça, Estação Experimental Alcides Carvalho, C.canephora cv. Apoatã, 06.iv.2001 e 02.iv.2002; 5+, Monte Alegre do Sul, Sítio São José, C. arabica cv. Mundo Novo, 14.i.2005, 02.ii.2005, 23.iii.2005, 13.iii.2005 e 01.v.2007; 4, Atibaia, Sítio Kurosawa, C. arabica cv. Mundo Novo, 19.xi.2007.

Relatos prévios no Brasil: Espírito Santo, Pernambuco, Rio Grande do Sul (Chiavegato 1975, Flechtmann \& Arleu 1984, Lorezato 1987, Lorenzato et al. 1986, Barbosa et al., 2003, Hernandes \& Feres 2006, Reis \& Zacarias 2007, Moraes \& Flechtmann, 2008).

\section{Steneotarsonemus sp.}

Espécimes examinados: 1 , , Jeriquara, Fazenda Boa Esperança, C. arabica cv. Mundo Novo, 17.xi.2001; 1, Garça, Estação Experimental Alcides Carvalho, C.arabica cv. Mundo Novo, 15.ii.2002.

\section{Tarsonemus confusus Ewing}

Tarsonemus confusus Ewing, 1939:26; Beer, 1954: 1173; Smiley, 1969:221; Kaliszewski, 1993: 40; Lofego et al., 2005: 24.

Espécimes examinados: 14엉 Jeriquara, Fazenda Boa Esperança, C. arabica cv. Mundo Novo, 02.v.2001 e 11.vii.2001; 4+, Garça, Estação Experimental Alcides Carvalho, C. arabica cv. Mundo Novo, 03.i.2002, 03.vii.2002 e 19.xii.2002; C. canephora cv. Apoatã, 01.ii.2002; 1 , Monte Alegre do Sul, sítio São José, C. arabica cv. Mundo Novo, 24.xi.2005.

Relatos prévios no Brasil: Minas Gerais, São Paulo (Spongoski et al. 2005, Lofego et al. 2005).

\section{Tarsonemus sp.}

Espécimes examinados: 4†, Jeriquara, Fazenda Boa Esperança, C. arabica cv. Mundo Novo, 02.v.2001, 16.v.2001 e 06.vi.2003; 2 , Garça, Estação Experimental Alcides Carvalho, C. arabica cv. Icatu Amarelo, 06.ix.2002; 1 , Pindamonhangaba, Fazenda Santa Emília, C. arabica, 04.i.2004; 1 , Paranapuã, C. arabica, 25.iii.2004.

\section{Ununguitarsonemus sp.}

Espécime examinado: 1q, Garça, Estação Experimental Alcides Carvalho, C.arabica cv.Catuaí Amarelo, 07.vi.2001.

\section{TENUIPALPIDAE BERLESE, 1913}

\section{Brevipalpus obovatus Donnadieu, 1875}

Brevipalpus obovatus Donnadieu, 1875: 116; Mesa et al., 2009: 37.
Brevipalpus amicus Chaudhri, 1972: 65 - Mitrofanov \& Strunkova (1979).

Brevipalpus origanum Baker, Tuttle \& Abbatiello, 1975: 18 Welbourn et al. (2003).

Brevipalpus pereger Donnadieu, 1875: 117 - Pritchard \& Baker (1958).

Tenuipalpus bioculatus McGregor, 1914: 354 - McGregor (1949).

Tenuipalpus inornatus Banks, 1912: 97 - Pritchard \& Baker (1958).

Tenuipalpus pseudocuneatus Blanchard, 1940: 11 - Pritchard \& Baker (1952).

Espécimes examinados: 2 , Garça, Estação Experimental Alcides Carvalho, C. arabica cv. Icatu Vermelho, 03.v.2002 e C. arabica cv. Icatu Amarelo, 07.viii.2002.

Relatos prévios no Brasil: Bahia, São Paulo (Bitancourt 1955, Paschoal 1968, Flechtmann 1976, Trindade \& Chiavegato 1994, Tamai et al. 1997).

Brevipalpus phoenicis (Geijskes, 1939)

Tenuipalpus phoenicis Geijskes, 1939: 23.

Brevipalpus deleoni Pritchard \& Baker 1958: 234 - De Leon (1961)

Brevipalpus mcbridei Baker, 1949: 374 - Pritchard \& Baker (1952).

Brevipalpus papayensis Baker 1949: 375 - Pritchard \& Baker (1952).

Brevipalpus phoenicoides González 1975: 86 - Evans et al. (1993).

Brevipalpus yothersi Baker 1949: 373 - Pritchard \& Baker (1952).

Brevipalpus phoenicis Sayed, 1946: 99; Pritchard \& Baker, 1958: 233; De Leon, 1961: 48; González, 1975: 82; Baker et al., 1975:18; Flechtmann, 1976: 58; Baker \& Tuttle, 1987: 98; Feres, 2000:164; Demite \& Feres, 2005: 832; Furtado et al., 2005: 138; Mesa et al., 2009: 40; Hernandes \& Feres, 2006.

Espécimes examinados: 13 , São Paulo, Instituto Biológico, C. arabica, 05.xi.2003 e 27.ii.2008; 1 ․ Cândido Mota, Distrito Frutal do Campo, C. arabica, 10.iii.2004; 11엉 Campinas, IAC, C. canephora cv. Guarini, 02.ix.2003; C. liberica var. Dewevrei, 02.ix.2003; C. liberica var. Piatã, 02.ix.2003; Tietê, 3 , CATI, Obatã, 07.viii.2003; 2, Lutécia, C. arabica cv. Catuaí Vermelho, 10.iii.2004; 2 , Itirapuã, sítio Santo Antônio, Bourbon, 21.i.2004; 5 , , Monte Alegre do Sul, Pólo Regional do Leste Paulista, C. arabica, 12.viii.2003; 1 q, Piracicaba, ESALQ/ USP, C. arabica, 12.viii.2003; 3 , Jaguariúna, C. arabica,

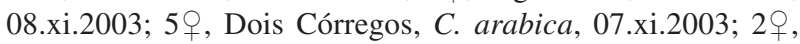
Itupeva, C. arabica, 26.ii.2004; 1 , Jeriquara, Fazenda Goretti, C. arabica cv. Sarchimor, 28.i.2005; 2 , , Monte Mor, Faz. Mundo Novo, C. arabica cv. Mundo Novo, 01.ix.2005; 11, Maracaí, Sítio Santa Margarida, C. arabica, 31.v.2006.

Relatos prévios no Brasil: Alagoas, Bahia, Ceará, Mato Grosso, Minas Gerais, Paraná, Pernambuco, Rio Grande do Sul, Rio de Janeiro e São Paulo (Amaral 1951, Flechtmann 1967, 1972 e 1976, Flechtmann \& Paschoal 1967, Flechtmann \& Arruda 1968, Paschoal \& Reis 1968, Vila \& Flechtmann 1970, Chagas 
1973, Flechtmann \& Abreu 1973, Moraes \& Flechtmann 1981, Flechtmann \& Abreu 1984, Lorenzato 1987, Pallini Filho et al. 1992, Lorenzato et al. 1986, Moraes et al. 1993, Trindade \& Chiavegato 1994, Rodrigues \& Nogueira 1996, Oliveira 1986, Reis et al. 2000, Feres 2000, Gondim Jr. 2000, Mineiro et al. 2001, Feres \& Nunes 2001, Zacarias 2001, Arruda Filho 2002, Ferla \& Moraes 2002, Barbosa et al. 2003, Daud \& Feres 2005, Spongoski et al. 2005, Demite \& Feres 2005, Furtado et al. 2005, Vasconcelos et al. 2005, Hernandes \& Feres 2006, Vis et al. 2006, Reis \& Zacarias, 2007, Moraes \& Flechtmann, 2008).

\section{Tenuipalpus sp.}

Espécime examinado: 1q, Jeriquara, Fazenda São Francisco, C. arabica cv. Catuaí Amarelo, 20.iii.2003.

\section{TETRANYCHIDAE DONNADIEU, 1875}

Aponychus sp.

Espécimes examinados: 2 ninfas, Jeriquara, Fazenda Boa Esperança, C. arabica cv. Mundo Novo, 22.ix.2001; 1 , Monte Alegre do Sul, sítio São José, C. arabica cv. Mundo Novo, 23.xii.2004.

Atrichoproctus sp.

Espécimes examinados: 2 , Jeriquara, Fazenda São Francisco, C. arabica cv. Mundo Novo, 08.iii.2002 e 06.v.2002; 1 \&, Garça, Estação Experimental Alcides Carvalho, C. arabica cv. Icatu Amarelo, 01.ii.2002.

Eotetranychus sp.

Espécimes examinados: 2 + , Jeriquara, Fazenda Boa Esperança,

C. arabica cv. Mundo Novo, 22.ix.2001 e 02.ix.2002.

Eutetranychus banksi (McGregor, 1914)

Tetranychus banksi McGregor, 1914: 358.

Anychus banksi (McGregor) McGregor, 1919: 644.

Eutetranychus banksi McGregor, 1950: 268; Pritchard \& Baker, 1955: 115; Flechtmann, 1967: 24; Flechtmann \& Baker, 1970: 156; Flechtmann \& Baker, 1975: 112; Bolland et al., 1998:75; Feres, 2000; Zacarias, 2001:97; Demite \& Feres, 2005:831; Hernandes \& Feres, 2006:9.

Espécimes examinados: 4 + e $1 \hat{\delta}$, Jeriquara, Fazenda Boa Esperança, C. arabica cv. Mundo Novo, 07.vi.2002, 2f,

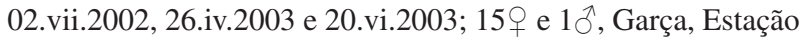
Experimental Alcides Carvalho, C. canephora cv. Apoatã, 06.vii.2001, 02.iv.2002, 03.v.2002, 03.vi.2002, 03.vii. 2002 e 30.iv.2003.

Relatos prévios no Brasil: Pernambuco, São Paulo (Flechtmann \& Baker 1975, Barbosa et al., 2003, Mineiro et al. 2004, Demite \& Feres 2005, Hernandes \& Feres 2006, Vis et al. 2006)

Mononychellus planki (McGregor, 1950)

Tetranychus planki McGregor, 1950: 300.

Eotetranychus planki (McGregor) Pritchard \& Baker, 1955.

Mononychellus planki Wainstein, 1971: 589; Flechtmann \& Baker, 1975: 117; Bolland et al., 1998: 92.

Espécimes examinados: 2 + e $1 \hat{\jmath}$, Jeriquara, Fazenda Boa Esperança, C. arabica cv. Mundo Novo, 16.ii.2002; Fazenda São Francisco, C. arabica cv. Mundo Novo, 15.ii.2002.

Relatos prévios no Brasil: Rio de Janeiro e São Paulo (Flechtmann \& Baker 1975).
Neotetranychus sp.

Espécime examinado: 1 ㅇ, Jeriquara, Fazenda Boa Esperança, C. arabica cv. Mundo Novo, 15.iii.2003.

Oligonychus ilicis (McGregor, 1917)

Tetranychus ilicis McGregor, 1917: 586.

Paratetranychus ilicis (McGregor) McGregor, 1919.

Oligonychus ilicis Pritchard \& Baker, 1955, 305; Flechtmann \& Baker, 1975: 117; Bolland et al., 1998:113.

Espécimes examinados: $106 \bigcirc$ e $6 \hat{\jmath}$, Jeriquara, Fazenda Boa Esperança, C. arabica cv. Mundo Novo, 02.ix.2002; Fazenda São Francisco, C. arabica cv. Mundo Novo, 06.v.2002; 1209 e $10 \hat{\jmath}$, Garça, Estação Experimental Alcides Carvalho, C. arabica cv. Icatu Vermelho, 08.vi.2001; 4 e e $2 \hat{\jmath}$, Jaboticabal, UNESP/ FCAV, C. arabica, 18.vii.2003.

Relatos prévios no Brasil: Minas Gerais, Paraná e São Paulo (Amaral 1951, Calza \& Sauer 1952, Flechtmann 1967, Heinrich 1972, Flechtmann \& Baker 1975, Mineiro et al. 2006, Reis \& Zacarias, 2007, Moraes \& Flechtmann, 2008).

Oligonychus yothersi (McGregor, 1914)

Tetranychus yothersi McGregor, 1914: 355.

Paratetranychus yothersi (McGregor) Pritchard \& Baker, 1955.

Oligonychus yothersi Pritchard \& Baker, 1955, 305; Flechtmann \& Baker, 1975: 118; Bolland et al., 1998:135.

Espécimes examinados: 36 q e $2 \hat{\jmath}$, Atibaia, Sítio Kurosawa, C. arabica cv. Catuaí Amarelo, 27.vi.2000, 2f, 11.vii.2000, 25.vii.2000; 11 + e 1ð̂, C. arabica cv. Mundo Novo, 20.viii.2007; $28 \bigcirc$ e $1 \widehat{\jmath}$, Monte Alegre do Sul, Sítio São José, 01.iv.2004, 05.x.2004, 16.iii.2005, 05.v.2006, 21.ii.2007 e 20.iii.2007.

Relatos prévios no Brasil: Minas Gerais, Paraná, Rio de Janeiro, São Paulo (Paschoal \& Reis 1968, Flechtmann \& Baker 1975, Gouveia et al. 2004, Pereira et al. 2005, Mineiro et al. 2008b).

\section{Oligonychus sp.}

Espécimes examinados: 1, São Paulo, Instituto Biológico, C. arabica, 05.xi.2003; 19, Campinas, IAC, C. canephora cv. Guarini, 02.ix.2003; 7올 Piracicaba, ESALQ/USP, C. arabica, 12.viii.2003; 6우, Dois Córregos, C. arabica, 07.xi.2003; 2 은, Itupeva, C. arabica, 26.ii.2004; 2 , Jeriquara, Fazenda Goretti, C. arabica cv. Sarchimor, 28.i.2005; 2 ㅇ, Monte Mor, Faz. Mundo Novo, C. arabica cv. Mundo Novo, 01.ix.2005.

Petrobia sp.

Espécimes examinados: 19 e 1 ninfa, Jeriquara, Fazenda Boa Esperança, C. arabica cv. Mundo Novo, 18.viii.2002 e 21.xii.2002.

Tetranychus sp. 1

Espécimes examinados: 19 , Campinas, IAC, C. canephora $\mathrm{cv}$. Guarini, 02.ix.2003; 2 , , Franca, Sítio Ripa, C. arabica cv. Catuaí Vermelho, 20.ii.2003; 2 , Monte Alegre do Sul, Pólo Regional do Leste Paulista, C. arabica cv. Mundo Novo, 07.vi.2004.

Tetranychus sp.2

Espécimes examinados: 2 , Jeriquara, Fazenda Boa Esperança, C. arabica cv. Mundo Novo, 22.ix.2001. 
TROMBIDIIDAE LEACH, 1815

Trombidium sp.

Espécimes examinados: $2 q$, Atibaia, Sítio Kurosawa, C. arabica cv. Mundo Novo, 20.vii.2007 e 19.xi.2007.

\section{TUCKERELLIDAE BAKER \& PRITCHARD, 1953}

Tuckerella pavoniformis (Ewing, 1922)

Tuckerella pavoniformis Ewing, 1922; Baker \& Pritchard, 1953: 253; Ochoa, 1989: 206.

Espécime examinado: 1 , Monte Alegre do Sul, Pólo Regional do Leste Paulista, C. arabica cv. Mundo Novo, 07.vi.2004.

Relatos prévios no Brasil: São Paulo (Mineiro \& Raga 2003, Mineiro et al. 2004).

\section{TYDEIDAE KRAMER, 1877}

\section{Lorryia formosa Cooremann, 1958}

Lorryia formosa Cooremann, 1958: 6; Baker, 1968: 995; Zacarias, 2001:63; Feres, 2000: 162; Feres et al., 2005: 5; Buosi et al., 2006: 11; Hernandes \& Feres, 2006:12; Demite et al., 2009:8.

Espécimes examinados: 1 , Tietê, CATI, C. arabica cv. Obatã, 07.viii.2003; 3요 Lutécia, C. arabica cv. Catuaí Vermelho, 10.iii.2004; 10, Campinas, IAC, C. liberica var. Piatã, 02.ix.2003; C. canephora cv. Apoatã, 26.x.2005; 9 , Piracicaba, ESALQ/USP, C. arabica, 12.viii.2003; C. canephora, 12.viii.2003; 4ㅇ, Jaguariúna, C. arabica, 08.xi.2003; 2ㅇ, Tatuí, Distrito de Morro do Alto, sítio Morro Alto, C. arabica, 08.i.2004; 1 , Paranapuã, C. arabica cv. Apoatã, 25.iii.2004; 6 , Jeriquara, Fazenda Boa Esperança, C. arabica cv. Mundo Novo, 27.vi.2001 e 25.vii.2001; 1 \%, Assis, Pólo Regional do Médio Paranapanema, C. arabica cv. Obatã, 05.ix.2003; 1 , Franca, Fazenda da Mata, C. arabica cv. Catuaí Vermelho,

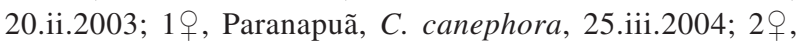
Mococa, Pólo Regional de Mococa, C. arabica var. Tupi, 18.vi.2004; 9 + , Atibaia, Sítio Kurosawa, C. arabica cv. Catuaí Amarelo, 13.vi.2000; 7우 Garça, Estação Experimental Alcides Carvalho, C. canephora cv. Apoatã, 06.iv.2001 e 09.v.2001; C. arabica cv. Icatu Amarelo, 01.iv.2002; cv. Mundo Novo, 06.vii.2001; 1 , , Monte Alegre do Sul, Sítio São José, C. arabica cv. Mundo Novo, 23.iii.2005; 1 \&, Pólo Regional do Leste Paulista, C. arabica sob mata, 01.xii.2006.

Relatos prévios no Brasil: São Paulo (Flechtmann 1973, Zacarias 2001, Mineiro \& Raga 2003, Mineiro et al. 2004, Daud \& Feres 2005, Spongoski et al. 2005, Demite \& Feres 2005).

\section{Lorryia sp.1}

Espécimes examinados: 19 , Jaguariúna, C. arabica, 08.xi.2003; 29, Atibaia, Sítio Kurosawa, C. arabica cv. Catuaí Amarelo, 11.vii.2000 e 25.vii.2000; 2 , Garça, Estação Experimental Alcides Carvalho, C.arabica cv. Mundo Novo, 13.vi.2003; cv. Icatu Amarelo, 29.i.2003; 10우, Jeriquara, Fazenda Boa Esperança, C. arabica cv. Mundo Novo, 25.vii.2001; 2 , Jaboticabal, UNESP/FCAV, C. arabica, 18.vii.2003; 5q, São Paulo, IB, C. arabica cv. Mundo Novo, 27.ii.2008; 1 † Monte Alegre do Sul, Sítio São José, C. arabica cv. Mundo Novo, 14.vi.2006.

\section{Lorryia sp.2}

Espécime examinado: 1 9 , Garça, Estação Experimental Alcides Carvalho, C. arabica cv. Mundo Novo, 07.viii.2001.
Lorryia sp.3

Espécimes examinados: 4일 Garça, Estação Experimental Alcides Carvalho, C. arabica cv. Mundo Novo, 03.i.2002; cv. Catuaí Amarelo, 06.vii.2001, 05.ix.2001 e 10.x.2001; 1웅, Jeriquara, Fazenda Boa Esperança, C. arabica cv. Mundo Novo, 20.vi.2003; 2 + , Fazenda São Francisco, C. arabica cv. Catuaí Amarelo, 23.ii.2002 e 20.vi.2003; 2q, Monte Alegre do Sul, Sítio São José, C. arabica cv. Mundo Novo, 30.xii.2004 e 02.ii.2005.

Lorryia sp.4

Espécime examinado: 1 + , Monte Alegre do Sul, Sítio São José, C. arabica cv. Mundo Novo, 12.xii.2007.

Pretydeus sp.

Espécimes examinados: 1ㅇ, Garça, Estação Experimental Alcides Carvalho, C. arabica cv. Mundo Novo, 22.vi.2001; 2 을 Atibaia, Sítio Kurosawa, C. arabica cv. Mundo Novo, 23.vii.2007 e 19.xi.2007.

Pseudolorryia sp.

Espécime examinado: 19 , Atibaia, Sítio Kurosawa, C. arabica cv. Mundo Novo, 23.iv.2007, 1f e 18.ii.2008.

Tydeus aff. costensis

Espécimes examinados: 2 , Jeriquara, Fazenda Boa Esperança, C. arabica cv. Mundo Novo, 13.vi.2001.

Tydeus sp.

Espécime examinado: 1, Monte Alegre do Sul, Sítio São José, C. arabica cv. Mundo Novo, 03.i.2008.

\section{Discussão}

Poucos levantamentos sobre a acarofauna de cafeeiros foram realizados em São Paulo. O primeiro deles foi realizado a partir da década de 1950 e que considerou apenas a ocorrência de $O$. ilicis e de B. phoenicis em vários municípios do Estado de São Paulo (Amaral 1951, Calza \& Sauer 1952) e posteriormente por Flechtmann (1967). Depois disso, levantamentos foram realizados apenas no início do século XXI (Mineiro et al. 2001, 2006a, b, 2008b). O presente estudo fez um levantamento de ácaros abrangendo, de modo geral, a ordem Trombodiformes nesta cultura em diversas regiões do Estado de São Paulo. Isto explicaria a dificuldade de identificar a maioria destes ácaros, uma vez que, muitas das espécies encontradas são novas para a ciência. Por esta razão, levantamentos de ácaros nessa cultura são importantes, porque permitem visualizar a grande diversidade e riqueza de espécies que ainda são desconhecidas nesta cultura. Estudos quanto a biologia e predação poderão servir de base para programas de manejo de ácaros predadores nesta cultura.

\section{Agradecimentos}

À FAPESP (Fundação de Amparo à Pesquisa do Estado de São Paulo) pela bolsa de pós-doutorado ao primeiro autor (Processo \# 2006/05798-7); aos Professores Dr. Carlos H.W. Flechtmann e Dr. Reinaldo J. F. Feres pela confirmação dos gêneros e espécies de Tetranychidae; à Dra. Denise Navia pela confirmação dos gêneros e espécies de eriofiídeos; ao Dr. Antonio C. Lofego pela confirmação dos gêneros de Tarsonemidae; ao Dr. Fábio A. Hernandes pela confirmação das espécies de Bdellidae; ao Dr. Eddie Ueckermann pela confirmação da espécie de Anystidae. 


\section{Referências Bibliográficas}

AMARAL, J.P. 1951. A infestação de ácaros nos cafezais. Biológico 17(7):130.

ARRUDA FILHO, G.P. 2002. Ácaros de Arecaceae da Mata Atlântica do Estado de São Paulo, com ênfase na família Stigmaeidae (Acari: Raphignathoidea). Dissertação de mestrado, Escola Superior de Agricultura "Luiz de Queiroz", Universidade de São Paulo, Piracicaba, $88 \mathrm{p}$.

ARRUDA FILHO, G.P. \& MORAES, G.J. 2003. Stigmaeidae mites (Acari: Raphignathoidea) from Arecaceae of the Atlantic forest in São Paulo State, Brazil. Neotrop. Entomol. 32(1):49-57.

BAKER, E.W. 1949. The genus Brevipalpus (Acarina: Pseudoleptidae). Am. Midl. Nat. 42:350-402.

BAKER, E.W. 1968. The genus Lorryia. Ann. Entomol. Soc. Amer. 61(4):986-1008.

BAKER, E.W. \& PRITCHARD, A.E. 1953. The family categories of tetranychoid mites, with a review of the new families Linotetranidae and Tuckerellidae. Ann. Entomol. Soc. Amer. 46 (2):243-258.

BAKER, E.W. \& TUTTLE, D.M. 1987. The false spider mites of Mexico (Tenuipalpidae: Acari). Tech. Bull./ U. S. Dep. Agric. 1706:1-237.

BAKER, E.W., TUTTLE, D.M., ABBATIELLO, M.J. 1975. The false spider mites of Northwestern and north central Mexico (Acarina: Tenuipalpidae). Smiths. Contrib. Zool. 194:1-22.

BANKS, N. 1904. Class III, Arachnida, Order I, Acarina, four new species of injurious mites. J. New York Entomol. Soc. 12:53-56.

BANKS, N. 1912. New American mites. Proc. Entomol. Soc. Wash. 14:96102.

BARBOSA, D.G.F., GONDIM Jr., M.G.C., BARROS, R. \& OLIVEIRA, J.V. 2003. Diversidade de ácaros em aceroleira (Malpighia emarginata A.DC.) na Universidade Federal de Pernambuco em Recife, PE. Neotrop. Entomol. 32(4):577-583.

BEER, R.E. 1954. A revision of the Tarsonemidae of the Western Hemisphere (Order Acarina). Univ. Kansas Sci. Bull. 36, 2(16):1091-1387.

BEER, R.E. \& NUCIFORA, A. 1965. Revisione dei generi della famiglia Tarsonemidae (Acarina). Boll. Zool. Agr. Bachicoltura, 7(2):19-43.

BITANCOURT, A.A. 1955. Estudos sobre a leprose dos citros I - Distribuição geográfica e sintomatologia. Arq. Inst. Biol. 22:161-184.

BLANCHARD, E.E. 1940. Tres ácaros dañinos para los cultivos argentinos. Rev. Fac. Agron. Nac. Plata 24:11-18.

BOLLAND, H.R., GUTIERREZ, J. \& FLECHTMANN, C.H.W. 1998. World catalogue of the spider mite family (Acari: Tetranychidae). Leiden, Netherlands, 392p

BUOSI, R., FERES, R.J.F., OLIVEIRA, A.R., LOFEGO, A.C. \& HERNANDES, F.A. 2006. Ácaros plantícolas (Acari) da "Estação Ecológica de Paulo de Faria", Estado de São Paulo, Brasil. Biota Neotrop. 6 (1): http:// www.biotaneotropica.org.br/v6n1/pt/ abstract?article+bn02006012006. (último acesso em 18/09/2007).

CALZA, R. \& SAUER, H.F.G. 1952. A aranha vermelha dos cafezais. Biológico 18(12):201-208.

CHAGAS, C.M. 1973. A associação do ácaro Brevipalpus phoenicis (Geijskes) à mancha anular do cafeeiro. Biológico 39(9):229-232.

CHAUDRI, W.M. 1972. The genus Brevipalpus in Pakistan. I. Description of six new species and redescription of one species, with new records. Pakistan J. Zool. 4(1):53-68.

CHIAVEGATO, L.G. 1975. Flutuação de populações de ácaros na cultura algodoeira em algumas regiões do Estado de São Paulo. Bragantia 34(15):241-255

COOREMAN, J. 1958. Note et observations sur les acariens VII. Photic graeca $\mathrm{n}$. sp. (Acaridiae, Canestriniidae) et Lorryia formosa n. sp. (Stomatostigmata, Tydeidae). Bull. Inst. Royal Sci. Nat. Belg. 34(8):110 .
DAUD, R.D. \& FERES, R.J.F. 2005. Diversidade e flutuação populacional de ácaros (Acari) em Maeba fistulifera Mart. (Euphorbiaceae) de dois fragmentos de mata estacional semidecídua em São José do Rio Preto, SP. Neotrop. Entomol. 34(2):191-201

DEMITE, P.R. \& FERES, R.J.F. 2005. Influência de vegetação vizinha na distribuição de ácaros em seringal (Hevea brasiliensis Muell. Arg., Euphorbiaceae) em São José do Rio Preto, SP. Neotrop. Entomol. 34(5): 829-836.

DEMITE, P.R., FERES, R.J.F., LOFEGO, A.C. \& OLIVEIRA, A.R. 2009. Plant inhabiting mites (Acari) from the Cerrado biome of Mato Grosso State, Brazil. Zootaxa 2061:45-60.

De LEON, D. 1961. The genus Brevipalpus in México, part II (Acarina: Tenuipalpidae). Fla. Entomol. 44(1):41-52.

DONNADIEU, A.L. 1875. Recherches pour servir à l'histoire des Tétranyques. Thèses, Faculté des Sciences de Lyon, Ann. Soc. Linnean, 131p.

EWING, H.E. 1939. A revision of the mites of the subfamily Tarsoneminae of North America, the West Indies and Hawaiian Islands. Unit. Stat. Dep. Agric., Techn. Bulletin, 653:1-63.

EVANS, G., CROMROY, H.L. \& OCHOA, R. 1993. The Tenuipalpidae of Honduras (Tenuipalpidae: Acari). Fla. Entomol. 76(1):126-155.

FERES, R.J.F. 2000. Levantamento e observações naturalísticas da acarofauna (Acari, Arachnida) de seringueiras cultivadas (Hevea spp., Euphorbiaceae) no Brasil. Rev. Bras. Zool. 17(1):157-173.

FERES, R.J.F. \& NUNES, M.A. 2001. Ácaros (Acari, Arachnida) associados a euforbiáceas nativas em áreas de cultivo de seringueiras (Hevea brasiliensis Muell. Arg.; Euphorbiaceae) na região noroeste do estado de São Paulo, Brasil. Rev. Bras. Zool. 18(4):253-264.

FERES, R.J.F., LOFEGO, A.C. \& OLIVEIRA, A.R. 2005. Ácaros plantícolas (Acari) da "Estação Ecológica do Noroeste Paulista", Estado de São Paulo, Brasil. Biota Neotrop. 5(1): http://biotaneotropica.org.br/v5n1/pt/ abstract?article+bn00405012005 (último acesso em 18/09/2007).

FERLA, N.J. \& MORAES, G.J. de. 2002. Ácaros (Arachnida, Acari) da seringueira (Hevea brasiliensis Muell. Arg.) no Estado do Mato Grosso, Brasil. Rev. Bras. Zool. 19(3):867-888.

FERLA, N.J., MARCHETTI, M.M. \& GONÇALVES, D. 2007. Ácaros predadores (Acari) associados à cultura do morango (Fragaria sp, Rosaceae) e plantas próximas no Estado do Rio Grande do Sul. Biota Neotrop. 7 (2) http:www.biotaneotropica.org.br/v7n2/pt/ abstract?article+bn01807022007 (último acesso em 18/09/2007).

FLECHTMANN, C.H.W. 1967. Os ácaros do cafeeiro. An. Esc. Sup. Agric. "Luiz de Queiroz" 24:91-95.

FLECHTMANN, C.H.W. 1972. Nota sobre ácaros (Acari) atacando fruteira-do-conde (Rollinia sp). An. Esc. Sup. Agric. "Luiz de Queiroz" 29:155-157.

FLECHTMANN, C.H.W. 1973. Lorryia formosa Cooreman, 1958: um ácaro dos citros pouco conhecido no Brasil. Ciên. Cult. 25(12):1179-1181.

FLECHTMANN, C.H.W. 1976. Preliminary report on the false spider mites (Acari: Tenuipalpidae) from Brazil and Paraguay. Proc. Entomol. Soc. Wash. 78(1):58-64

FLECHTMANN, C.H.W. 1981. New records of mites from Brazil with description of two species in the genus Oligonychus Berlese (Acari, Tetranychidae). Rev. Bras. Biol. 41(4):861-866.

FLECHTMANN, C.H.W. \& PASCHOAL, A.D. 1967. Os ácaros dos citrus. Solo 2:53-56.

FLECHTMANN, C.H.W. \& ARRUDA, G.P. 1968. Breve nota sobre ácaros de plantas no Estado de Pernambuco. Rev. Bras. Entomol. 13:95-97.

FLECHTMANN, C.H.W. \& ARANDA, B.R. 1970. New records and notes on eriophyid mites from Brazil and Paraguay, with a list of Eriophyidae from South America. Proc. Entom. Soc. Wash. 72(1):94-98.

FLECHTMANN, C.H.W. \& ABREU, J.M. 1973. Ácaros fitófagos do Estado da Bahia, Brasil (Notas preliminares). Ciên. Cult. 25(3):244-251.

FLECHTMANN, C.H.W. \& ARLEU, R.J. 1984. Oligonychus coffeae (Nietner, 1861), um ácaro tetraniquídeo da seringueira (Hevea brasiliensis) novo para o Brasil e observações sobre outros ácaros desta planta. Ecossistema 9:123-125. 
FLECHTMANN, C.H.W. \& BAKER, E.W. 1975. A report on the Tetranychide (Acari) of Brazil. Rev. Bras. Entomol. 19(3):111-122.

FLECHTMANN, C.H.W. \& SANTANA, D.L.Q. 1997. A Preliminary note on mites on corn in Brazil with redescription of Catarhinus tricholaenae and Oligonychus zeae (Acari: Diptilomiopidae, Tetranychidae). Syst. Appl. Acarol. 2:189-194.

FRANCO, R.A., REIS, P.R., ZACARIAS,M.S., ALTOÉ, B.F., PEDRO NETO, M. 2008. Dinâmica populacional de Oligonychus ilicis (McGregor, 1917) (Acari: Tetranychidae) em cafeeiro e de fitoseídeos associados a ele. Coffee Science, 3(1):38-46.

FURTADO, I.P., KREITER, S., MORAES, G.J., TIXIER, M.S., FLECHTMANN, C.H.W. \& KNAPP, M. 2005. Plant mites (Acari) from Northeasthern Brazil, with descriptions of two new species of the family Phytoseiidae (Mesostigmata). Acarologia 14(2-3):131-143.

GEIJSKES, D.C. 1939. Beitraege zur Kenntnis der europaeischen Spinnmilben (Acari, Tetranychidae) mit besonderer Beruecksichtigung der niederlaendischen Arten. Meded. Landbouwhoogesch. Wagening. 42:1-68.

GONDIM JUNIOR, M.G.C. 2000. Ácaros de palmeiras (Arecaceae) en áreas dos Estados de São Paulo e Pernambuco. Tese de doutorado, Escola Superior de Agricultura "Luiz de Queiroz", Universidade de São Paulo, Piracicaba, 161p.

GONZÁLEZ, R.H. 1975. Revision of the Brevipalpus phoenicis "complex" with descriptions of new species from Chile and Thailand (Acarina: Tenuipalpidae). Acarologia 17(1):81-91.

GONZALEZ-RODRIGUES, R.H. 1965. A taxonomic study of the genera Mediolata, Zetzellia and Agistemus (Acarina: Stigmaeidae). Univ. Calif. Publ. Entomol. 41:1-64.

GOUVÊA, A., ZANELLA, C.F. \& ALVES, L.F.A. 2004. Dinâmica populacional do ácaro Oligonychus yothersi (McGregor), 1914, (Acari: Tetranychidae) em plantas de erva-mate Ilex paraguariensis ST. Hill. (Aqüifoliácea), em dois vizinhos, PR. Sci. Agr. Paran. 3(1):25-48.

HEINRICH, W.O. 1972. Contribuição ao estudo da biologia do Oligonychus (Oligonychus) ilicis (Acarina: Tetranychidae). Tese de Doutorado, Escola Superior de Agricultura "Luiz de Queiroz", Universidade de São Paulo, Piracicaba, 116p.

HERNANDES, F.A. \& FERES, R.J.F. 2005. Two new species of Zetzellia Oudemans (Acari: Stigmaeidae) that threaten the concept of genera: disgeneric marriage? Zootaxa 1048:27-44.

HERNANDES, F.A. \& FERES, R.J.F. 2006. Review about mites (Acari) of rubber trees (Hevea spp., Euphorbiaceae) in Brazil. Biota Neotropica, 6 (1): http://www.biotaneotropica.org.br/v6n1/pt/ abstracts?article+bn00406012006 (último acesso em 04/08/2006).

HERNANDES, F.A., DAUD, R.D. \& FERES, R.J.F. 2007. A new species of Hexabdella (Acari: Bdellidae) from Brazil. Zootaxa 1501:57-63.

HERNANDES, F.A., DAUD, R.D. \& FERES, R.J.F. 2008. Two new species of Bdellidae (Acari: Prostigmata) from Brazil. Intern. J. Acarol. 34(3):259-266.

JEPPSON, L.R., KEIFER, H.H. \& BAKER, E.W. 1975. Mites injurious to economic plants. University of California Press, 614p.

KALISZEWSKI, M. 1993. Key to Paleartic species of the genus Tarsonemus (Acari, Tarsonemidae). Wydawnictwo Naukowe Uniwersytet Im. Adama Mickiewica w Poznaniu, 14:1-204.

KEIFER, H.H. 1959. New eriophyid studies. Ann. Entomol. Soc. Amer. 52:649-657.

LINDQUIST, E.E. 1986. The world genera of Tarsonemidae (Acarina: Heterostigmata): A morphological, phylogenetic and systematic revision with a reclassification of family-group taxa in the Heterostigmata. Mem. Entomol. Soc. Canada 136:517.

LOFEGO, A.C.L., OCHOA, R. \& MORAES, G.J. 2005. Some tarsonemid mites (Acari: Tarsonemidae) from the Brazilian "Cerrado" vegetation, with descriptions of three new species. Zootaxa 823:1-27.

LORENZATO, D. 1987. Controle biológico de ácaros fitófagos na cultura da macieira no município de Farroupilha, RS. Agron. Sulriogr. 23(2):167-183.
LORENZATO, D., GRELLMANN, E.O., CHOUÈNE, E.C. \& MEYERCACHAPUZ, L.M. 1986. Flutuação populacional de ácaros fitófagos e seus predadores associados à cultura da macieira (Malus domestica Bork) e efeitos dos controles químico e biológico. Agron. Sulriogr. 22(2):215-242.

MATIOLI, A.L., UECKERMANN, E.A. \& OLIVEIRA, C.A.L. 2002. Some stigmaeid and eupalopsellid mites from citrus orchards in Brazil (Acari: Stigmaeidae and Eupalopsellidae). Intern. J. Acarol. 28(2):99-120.

McGREGOR, E.A. 1914. Four new tetranychids. Ann. Entomol. Soc. Amer. 7:354-364.

McGREGOR, E.A. 1917. Descriptions of seven new species of red spiders. Proc. U. S. Nat. Mus. 51:581-590.

McGREGOR, E.A. 1919. The red spiders of America and a few European species likely to be introduced. Proc. Nat. Mus. 56:641-679.

McGREGOR, E.A. 1949. Nearctic mites of the family Pseudoleptidae. Mem. South. Calif. Acad. Sciences 3 (2):1-45.

McGREGOR, E.A. 1950. Mites of the family Tetranychidae. Amer. Midd. Natur. 44 (2):257-420.

MESA, N.C.: OCHOA, R.: WELBOURN, W.C., EVANS, G.A. \& MORAES, G.J. 2009. A catalog of the Tenuipalpidae (Acari) of the World with a key to genera. Zootaxa, 2098:1-185.

MEYER, M.K.P (SMITH) \& UECKERMANN, E.A. 1989. African Rhaphignathoidea (Acari: Prostigmata). Entomol. Mem. Dept. Agric. Water Supp. Repub. S. Africa, n. 74, 58p.

MINEIRO, J.L.C. \& RAGA, A. 2003. Ocorrência de ácaros (Arachnida: Acari) em plantas de lichia (Litchi chinensis Sonn) no Estado de São Paulo. Arq. Inst. Biol. 70 (Supl.):1-4.

MINEIRO, J.L.C., RAGA, A. \& LOFEGO, A.C. 2004. Ocorrência de ácaros (Arachnida: Acari) em aceroleira (Malpighia emarginata A.DC.) no Estado de São Paulo. Arq. Inst. Biol. 71 (Supl.): 1-4.

MINEIRO, J.L.C., SATO, M.E., RAGA, A., SOUZA FILHO, M.F., SILOTO, R.C., MORAES, G.J. \& SPONGOSKI, S. 2001. Distribuição da acarofauna em cafeeiro (Coffea arabica var. Catuaí Amarelo) em Atibaia, SP. In II Simpósio de Pesquisa dos cafés do Brasil. Vitória, ES, p.1471-1474.

MINEIRO, J.L.C., SATO, M.E., RAGA, A., ARTHUR, V., MORAES, G.J., SARRETA, F.O. \& CARRIJO, A. 2006a. Diversidade de ácaros (Arachnida: Acari) em Coffea arabica L. cv. Mundo Novo, nos municípios de Jeriquara e Garça, Estado de São Paulo. Biota Neotropica, 6 (2): http:// www.biotaneotropica.org.br/v6n2/pt/abstracts?article+bn01106062006 (último acesso em 03/09/2006).

MINEIRO, J.L.C., SATO, M.E., RAGA, A., ARTHUR, V., CANGANI, K.G. \& BARBOSA, F.V. 2006b. Diversidade de ácaros (Arachnida: Acari) em cinco cultivares de duas espécies de cafeeiros (Coffea spp.) em Garça, Estado de São Paulo. Arq. Inst. Biol. 73(3):333-341.

MINEIRO, J.L.C., SATO, M.E., RAGA, A., ARTHUR, V. 2008a. Population dynamics of phytophagous and predaceous mites on coffee in Brazil, with emphasis on Brevipalpus phoenicis (Acari: Tenuipalpidae). Exp. App. Acarol. 44:277-291.

MINEIRO, J.L.C., SATO, M.E., RAGA, A., SOUZA FILHO, M.F. \& SPONGOSKI, S. 2008b. Incidência de ácaros em cafeeiro cv. Catuaí Amarelo. Bragantia, 67(1):197-201.

MITROFANOV, V.I., STRUNKOVA, Z.I. 1979. A key to false spider mites. Operdelitl'Kleshchei-ploskotelok. 148:1-148.

MORAES, G.J., ALENCAR, J.A., LIMA, J.L.S., YANINEK, J.S. \& DELALIBERA Jr., I. 1993. Alternative plant habitats for common phytoseiid predators of cassava green mite (Acari: Phytoseiidae, Tetranychidae) in Northeast Brazil. Exp. Appl. Acarol. 17:77-90.

MORAES, G.J. \& FLECHTMANN, C.H.W. 1981. Ácaros fitófagos do nordeste do Brasil. Pesq. Agrop. Bras. 16 (2):177-186.

MORAES, G.J. \& FLECHTMANN, C.H.W. 2008. Manual de acarologia: acarologia básica e ácaros de plantas cultivadas no Brasil. Holos Editora, Ribeirão Preto, SP, 308p.

OCHOA, R. 1989. The genus Tuckerella in Costa Rica (Acari: Tuckerellidae). Intern. J. Acarol. 15 (4):205-207. 
OLIVEIRA, C.A.L. 1986. Flutuação populacional e medidas de controle do ácaro da leprose Brevipalpus phoenicis (Geijskes, 1939) em citros. Laranja 7:1-31.

OTTO, J. C. 1999. Revision of the genus Erythracarus Berlese (Acarina: Anystidae: Erythracarinae). J. Nat. Hist. 33(6):825-909.

PALLINI FILHO, A., MORAES, G.J. \& BUENO, V.H.P. 1992. Ácaros associados ao cafeeiro (Coffea arabica L.) no sul de Minas Gerais. Ciên. Prát. 16:303-307.

PASCHOAL, A.D. 1968. Espécies de ácaros encontrados em plantas no Estado de São Paulo. Ciên. Cult. 20 (2):258

PASCHOAL, A.D. \& REIS, P.R. 1968. Relação de ácaros encontrados em plantas. Rev. Agric. 43(3-4):137-139.

PEREIRA, F.F., ANJOS, N., ALMADO, R.P., RODRIGUES, L.A.L. 2005. Primeiro registro de Oligonychus yothersi (McGregor) (Acari: Tetranychidae) em Eucalyptus grandis Hill Ex Maiden no Brasil. Rev. Árvore 29(4):657-659.

PRITCHARD, A.E. \& BAKER, E.W. 1952. The false spider mites of California (Acarina: Phytoptipalpidae). Public. Entomol. 9(1):1-94.

PRITCHARD, A.E. \& BAKER, E.W. 1955. A revision of the spider mite family Tetranychidae. Pac. Coast Entomol. Soc., Mem. Ser. Vol. 2, 472p.

PRITCHARD, A.E. \& BAKER, E.W. 1958. The false spider mites (Acarina: Tenuipalpidae). Univ. Calif. Publ. Entomol. 14:175-274.

REIS, P.R., SOUZA, J.C., SOUZA, E.O. \& TEODORO, A.V. 2000. Distribuição espacial do ácaro Brevipalpus phoenicis (Geijskes) (Acari: Tenuipalpidae) em cafeeiro (Coffea arabica L.). An. Soc. Entomol. Brasil 29(1):177-183.

REIS, P.R. \& ZACARIAS, M.S. 2007. Ácaros em cafeeiros. EPAMIG, Boletim Técnico, 81, 76p.

RODRIGUES, J.C.V., NOGUEIRA, N.L. 1996. Ocorrência de Brevipalpus phoenicis G. (Acari: Tenuipalpidae) em Ligustrum lucidum (Oleaceae) associado à mancha-anelar do ligustre. An. Soc. Entomol. Brasil 25(2):343-344

SAYED, M.T. 1946. Description of Tenuipalpus granati nov. spec. and Brevipalpus pyri nov. spec. (Acarina: Trichadenidae). Bull. Soc. Fouad I Entomol. 30:99-103.
SMILEY, R.L. 1967. Some Tarsonemidae from the Republic of the Congo (Acarina). Proc. Entomol. Soc. Wash. 66:145-150.

SMILEY, R.L. 1969. Further studies on the Tarsonemidae, II (Acarina). Proc. Entomol. Soc. Wash. 71 (2):218-229.

SPONGOSKI, S., REIS, P.R. \& ZACARIAS, M.S. 2005. Acarofauna da cafeicultura de cerrado em Patrocínio, Minas Gerais. Ciênc. Agrotéc. 29(1):9-17.

SUMMERS, F.M. 1960. Eupalopsis and eupalopsellid mites (Acarina: Stigmaeidae, Eupalopsellidae). Fla. Entomol. 43(3):119-138.

TAMAI, M.A., MORAES, G.J., SILVA, C.A.D., NUNES, A.M. 1997. Suitability of Brevipalpus obovatus as prey to Neoseiulus idaeus (Acari: Tenuipalpidae, Phytoseiidae) on cassava. Syst. Appl. Acarol. 2:101106.

TRINDADE, M.L.B., CHIAVEGATO, L.G. 1994. Caracterização biológica dos ácaros Brevipalpus obovatus D., B. californicus B. e B. phoenicis G. (Acari: Tenuipalpidae). An. Soc. Entomol. Brasil 23(2):189-195.

VASCONCELOS, G.J., SILVA, F.R., BARBOSA, D.G.F., GONDIN JUNIOR, M.G.C., MORAES, G.J. 2005. Ocorrência de Eriophyoidea, Tenuipalpidae, Tarsonemidae e Tukerellidae (Acari) em fruteiras no Estado de Pernambuco, Brasil. Caatinga 18(2):98-104.

VILA, W.M. \& FLECHTMANN, C.H.W. 1976. Ácaros em essências florestais. Silvic. São Paulo 7:99-102.

VIS, R.M.J., MORAES, G.J. \& BELLINI, M.R. 2006. Mites (Acari) of rubber trees (Hevea brasiliensis Muell. Arg., Euphorbiaceae) in Piracicaba, State of São Paulo, Brazil. Neotrop. Entomol. 35(1):112-120.

WAINSTEIN, B.A. 1971. Mononychellus, a new name for Mononychus (Acariformes, Tetranychidae). Zool. Zh. 50(4):589.

WALTER, D.E. \& KRANTZ, G.W. 2009. Collecting, rearing, and preparing specimens. In: Krantz \& Walter (Eds.). A manual of Acarology. $3^{\text {rd }}$ ed. Texas Tech Univesity Press, USA, 807p.

WELBOURN, W.C., OCHOA, R., KANE, E.C., ERBE, E.F. 2003. Morphological observations on Brevipalpus phoenicis (Acari: Tenuipalpidae) including comparisons with $B$. californicus and $B$. obovatus. Exp. App. Acarol. 30(1/3):107-133.

ZACARIAS, M.S. 2001. Diversidade de ácaros (Arthropoda: Acari) em euforbiáceas (Euphorbiaceae) de três localidades do Estado de São Paulo. Tese de Doutorado, Escola Superior de Agricultura, Universidade de São Paulo, Piracicaba, 154p. 
